Research Article

\title{
Optimal Energy Control Strategy Design for a Hybrid Electric Vehicle
}

\author{
Yuan Zou, Hou Shi-jie, Li Dong-ge, Gao Wei, and Xiao-song Hu \\ National Engineering Laboratory for Electric Vehicles, School of Mechanical Engineering, Beijing Institute of Technology, \\ Beijing 100081, China
}

Correspondence should be addressed to Yuan Zou; zouyuan@bit.edu.cn

Received 8 September 2012; Revised 3 December 2012; Accepted 25 December 2012

Academic Editor: Geert Wets

Copyright (c) 2013 Yuan Zou et al. This is an open access article distributed under the Creative Commons Attribution License, which permits unrestricted use, distribution, and reproduction in any medium, provided the original work is properly cited.

A heavy-duty parallel hybrid electric truck is modeled, and its optimal energy control is studied in this paper. The fundamental architecture of the parallel hybrid electric truck is modeled feed-forwardly, together with necessary dynamic features of subsystem or components. Dynamic programming (DP) technique is adopted to find the optimal control strategy including the gear-shifting sequence and the power split between the engine and the motor subject to a battery SOC-sustaining constraint. Improved control rules are extracted from the DP-based control solution, forming near-optimal control strategies. Simulation results demonstrate that a significant improvement on the fuel economy can be achieved in the heavy-duty vehicle cycle from the natural driving statistics.

\section{Introduction}

Commercial transport vehicles, especially heavy-duty trucks, play an important role in the socialism construction of China. More than a half of the freight transported in China is carried by heavy-duty trucks. The increasing number of merely gasoline/diesel-propelled trucks brings some negative impacts, such as excessive fuel consumption and severe air pollution. In order to reduce the dependence on carbonbased fuel and poisonous emissions, hybrid powertrain has been widely studied recently. Owing to the dual-powersource nature, the complex configuration, and the operation modes, the control strategy of hybrid electric vehicle (HEV) is typically more complicated than that of traditional enginebased vehicle. Therefore, system-level vehicle simulation methodology is often applied to implement accurate sizing and matching studies, as well as to develop effective energy control algorithms, before the final design and physical prototyping.

The existing energy management and control strategies of hybrid vehicle can be mainly classified into three categories. The first type employs heuristic control techniques, such as rules/fuzzy logic for the control algorithm development $[1,2]$. The principle is commonly based on the concept of "load leveling," which attempts to operate the internal combustion engine in an efficient area and the battery as a load-leveling device used to provide the remaining power demand.

The second type of approach is based on static optimization methods that instantaneously determine the efficient power split between different energy sources by minimizing a cost function. To calculate the cost of energy, the electric energy is translated into an equivalent amount of fuel $[3,4]$. Due to its relatively simple point-wise optimization nature, it is possible to extend the optimization scheme to solve the simultaneous fuel economy and emission optimization problem [5].

The fundamental mechanism of the third type of HEV control algorithm considers the dynamic nature of the vehicle system when performing an optimization [6-8]. A time horizon is involved in this type of dynamic optimization, instead of a time instant in the static one mentioned above. Power split algorithms obtained from dynamic optimization are thus more accurate under transient conditions but are computationally more intensive. Despite that dynamic optimization cannot be realized onboard due to its preview nature and heavy computation requirement, it has been recognized as a good benchmark to the first two types of algorithms.

The main purpose of this paper is to use dynamic programming (DP) to solve the optimal control problem of a heavy-duty hybrid truck. We thoroughly analyze and discuss 


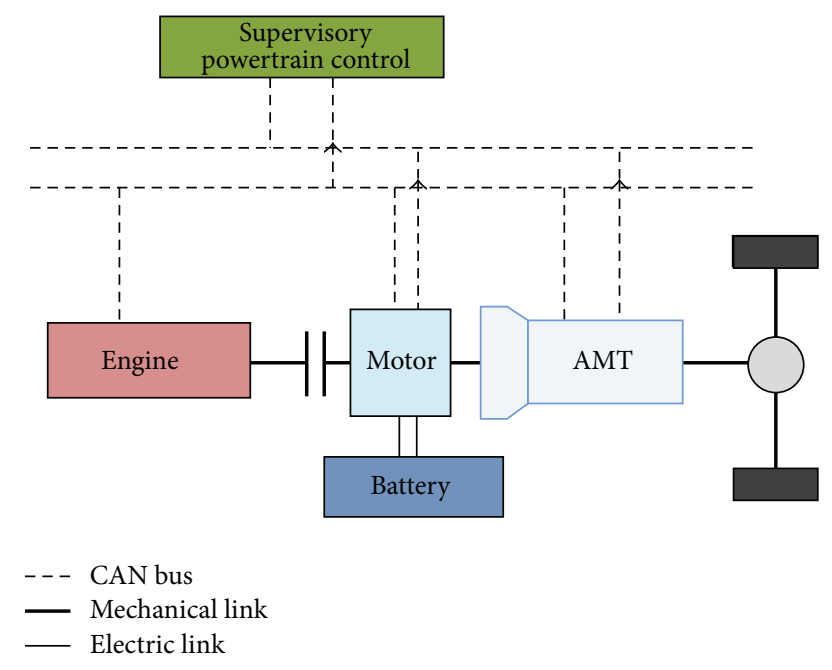

FIGURE 1: Architecture of the hybrid electric truck.

the DP-based results, from which the real-time control rules are extracted. A feed-forward simulation scheme is employed so as to enable the study of vehicle energy control strategy under realistic transient conditions. It can be found that the performance of the developed rule-based algorithm can be improved significantly relative to that based on static optimization.

The remainder of this paper is organized as follows. In Section 2, a heavy-duty hybrid electric truck model is built, followed by an explanation of the preliminary rulebased control strategy. The DP procedure and corresponding optimization problem are introduced in Section 3. In Section 4, the accomplished DP-based results and the realtime control rules are depicted, and the fuel economy results of the developed control strategy are also evaluated. The conclusions are presented in Section 5.

\section{Hybrid Electric Truck Model}

2.1. Vehicle Configuration. The vehicle architecture is given in Figure 1. The truck has a pretransmission parallel hybrid configuration allowing for a smaller electric motor/generator, an easier packing, and reduced spin losses, compared to the posttransmission type [9]. The diesel engine WP7.210 was manufactured by Weichai Power Co., Ltd. In order to ensure the accessibility of the total peak power, a $90 \mathrm{~kW}$ electric motor/generator was selected, and a $60 \mathrm{Ah}$ lithiumion battery was chosen as the onboard energy storage. A 9gear-automated mechanical transmission (AMT) was used. The parameters of the vehicle and its components are listed in Table 1.

A system-level HEV model is developed in the MAT$\mathrm{LAB} /$ Simulink environment, as shown in Figure 2. The HEV model is an accurate but moderate model appropriate for efficient evaluation of fuel economy [10]. Some fast dynamics, like intake manifold filling and motor dynamics, are much quicker than energy dynamics and are thus neglected.
TABLE 1: Parameters of the hybrid electric truck.

\begin{tabular}{ll}
\hline DI Diesel Engine & $7.0 \mathrm{~L}, 155 \mathrm{kw}$ at $2000 \mathrm{rpm}, 900 \mathrm{Nm}$ at \\
& 1300-1600 rpm \\
& Maximum power: $90 \mathrm{kw}$ \\
Motor/Generator & Maximum torque: $600 \mathrm{~N}-\mathrm{m}$ \\
& Maximum speed: $2400 \mathrm{rpm}$ \\
& Capacity: $60 \mathrm{Ah}$ \\
Lithium-ion & Number of modules: 25 \\
Battery & Nominal voltage: 12.5 (volts/module) \\
& 9 speed, \\
AMT & GR: $12.11 / 8.08 / 5.93 / 4.42 / 3.36 / 2.41 / 1.76 / 1.32 / 1$ \\
Vehicle & Curb weight: $16000 \mathrm{~kg}$ \\
\hline
\end{tabular}

2.2. Preliminary Rule-Based Control Strategy. The preliminary rule-based control strategy is designed based on the static optimization algorithm minimizing the total equivalent fuel consumption - sum of the fuel consumption and battery energy consumption rates at every step. The equivalent fuel consumption cost is defined as [11]

$$
m_{f_{\text {_total }}}=m_{f_{\text {_eng }}}+m_{f_{\text {_elec }}},
$$

where $m_{f_{\text {_eng }}}$ and $m_{f_{- \text {elec }}}$ represent the fuel consumptions of the engine and electric machines, respectively. $m_{f_{-} \text {elec }}$ can be calculated using the following equation:

$$
m_{f_{\text {elec }}}=\lambda \cdot \Delta t \cdot \frac{P_{\text {elec }}}{\eta_{\text {trans }}},
$$

where $\lambda$ is a conversion factor from electrical energy to engine fuel consumption, and its value was specified as $3.3 e-5$. $\Delta t=1 \mathrm{~s}$ is the time step. $P_{\text {elec }}$ represents the power of electric machines. $\eta_{\text {trans }}=\eta_{\text {batt }} \cdot \eta_{i} \cdot \eta_{\text {MG }}$ is the total efficiency of the electrical system composed of the battery, inverter, and motor/generator. The optimal control $u(k)$ can be obtained by solving the following equation:

$$
\text { Minimize } \underset{u(k)}{J}=\int m_{f_{\text {-total }}} d t
$$

Here, the control variables include the engine throttle and the transmission gear number, which can be solved using the following function written in the form of .m file in MATLAB

$$
\text { [throt_opt, gear_opt] = optimal (shf_spd, req_power), }
$$

where throt_opt and gear_opt are the optimal engine throttle and transmission gear, respectively, shf_spd is the final drive input shaft speed, and req-power is the power requested.

2.3. Fuel Economy Evaluation. A nature driving schedule proposed by Weichai Power is shown in Figure 3. The battery SOC correction procedure proposed in $[12,13]$ was used to correct fuel economy, when the initial and final battery SOC values are not identical. The hybrid electric truck, given the preliminary rule-based control strategy, achieved a fuel economy of 6.78 miles per gallon (MPG). However, this rulebased control strategy is component based rather than system based. Hence, this control strategy is not globally optimal. This motivates the use of DP as an analysis and design tool. 


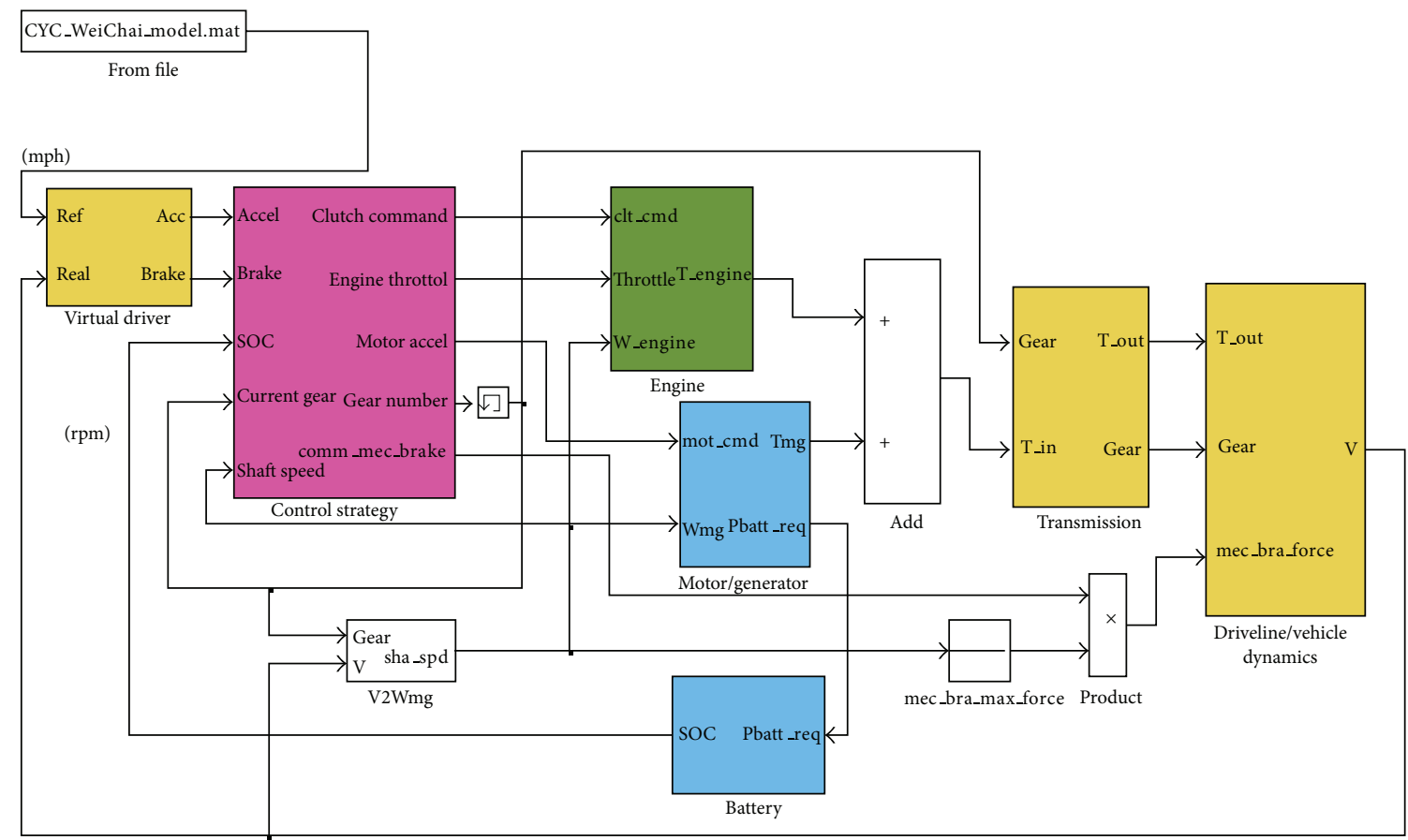

FIGURE 2: HEV powertrain model in Simulink.

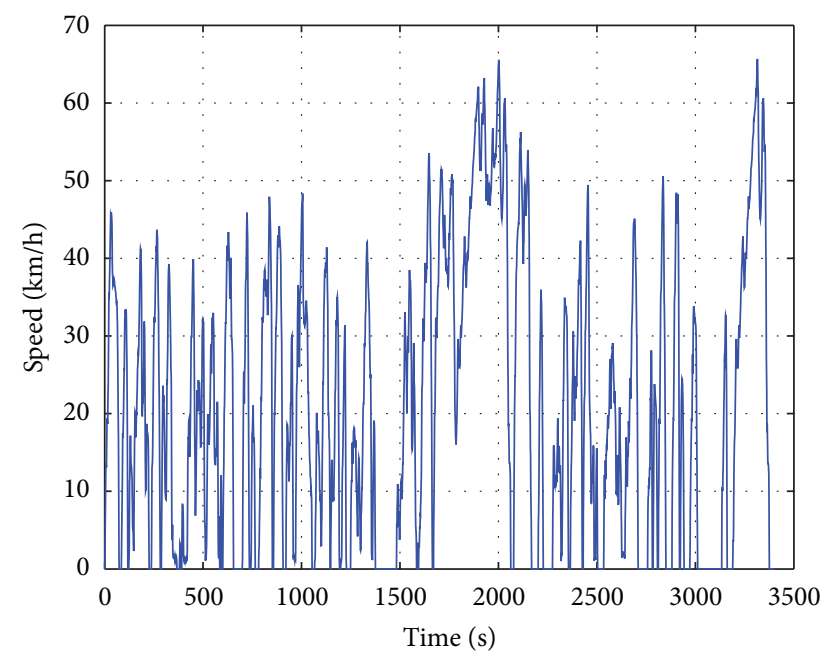

- Weichai heavy duty cycle

FIGURE 3: Nature driving schedule from Weichai Power Co., Ltd.

\section{Dynamic Programming Problem}

DP is a powerful tool for solving dynamic optimization problems, due to its guaranteed global optimality even for nonlinear dynamic systems with constraints. Given a driving cycle, the DP-based algorithm can obtain the optimal operating strategy minimizing the cost with subject to the diverse constraints.

3.1. Model Simplification. Since the system-level dynamics are the main concern of evaluating fuel economy over a long

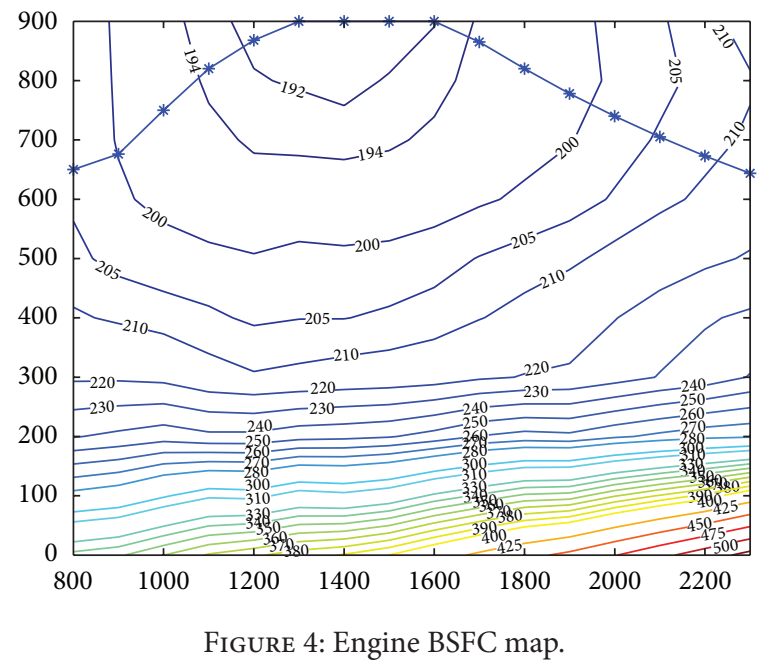

driving cycle, the dynamics faster than $1 \mathrm{~Hz}$ are neglected in the model used by DP. Analysis of the dynamic modes determines that only two state variables of the model are needed: the transmission gear number and battery SOC. The engine throttle and the transmission gear shifting command were chosen to be the control variables. The simplifications of the main sub systems are described as follows.

3.1.1. Engine. The engine dynamics are ignored based on the quasi-static assumption [14]. The fuel consumption is a static function of two independent variables: engine speed and engine torque. The engine map is shown in Figure 4. Herein, we assume that the engine is fully warmed up such that engine temperature effect is not considered. 


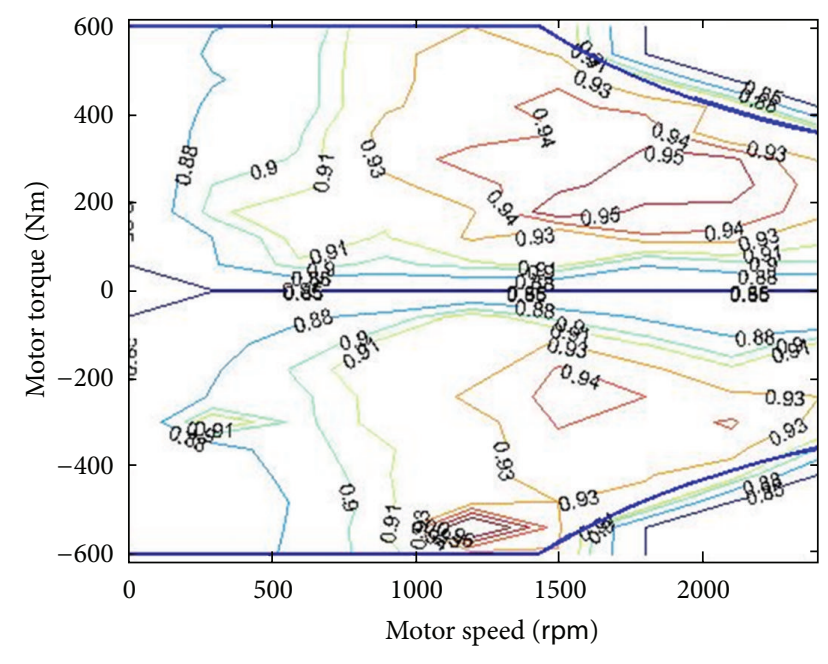

FIGURE 5: Motor efficiency map.

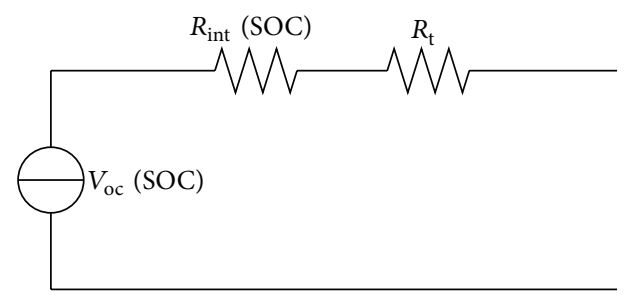

FIGURE 6: Internal resistive battery model.

3.1.2. Motor. Similar to the engine, the motor model was established using the experimental data. The motor efficiency is a function of motor torque and speed $\eta_{m}=f\left(T_{m}, \omega_{m}\right)$, as shown in Figure 5. Considering the battery power and motor torque limit, the final motor torque becomes

$$
T_{m}= \begin{cases}\min \left(T_{m, \text { req }}, T_{m, \text { dis }}\left(\omega_{m}\right)\right), & \text { if } T_{m, \text { req }}>0, \\ \max \left(T_{m, \text { req }}, T_{m, \text { chg }}\left(\omega_{m}\right)\right), & \text { if } T_{m, \text { req }}<0,\end{cases}
$$

where $T_{m, \text { req }}$ is the requested motor torque; $T_{m \text {,dis }}$ and $T_{m, \text { chg }}$ are the maximum motor torques in the motoring and charging modes, respectively.

3.1.3. Battery. A $20 \mathrm{kWh}$ battery was selected for the target truck, and the equivalent circuit battery model from ADVISOR package was adopted. The schematic diagram of the equivalent circuit model is shown in Figure 6. We ignored the thermal effects and transients so that the only state in the battery model is the SOC as follows:

$$
\begin{aligned}
& \operatorname{SOC}(k+1) \\
& =\operatorname{SOC}(k) \\
& \quad-\left(V_{\mathrm{oc}}-\sqrt{V_{\mathrm{oc}}^{2}-4\left(R_{\mathrm{int}}+R_{t}\right) \cdot T_{m} \cdot \omega_{m} \cdot \eta_{m}^{-\operatorname{sgn}\left(T_{m}\right)}}\right) \\
& \quad \times\left(2\left(R_{\mathrm{int}}+R_{t}\right) \cdot Q_{\max }\right)^{-1},
\end{aligned}
$$

where both the internal resistance $R_{\mathrm{int}}$ and the open circuit voltage $V_{\text {oc }}$ are a function of the battery SOC. $Q_{\max }$ is the maximum battery charge, and $R_{t}$ is the terminal resistance.

3.1.4. Driveline. The driveline is defined as the system from the transmission input shaft to the wheel. The following equations are used to describe the transmission and final drive gear models:

$$
\begin{gathered}
T_{\text {wheel }}=\eta_{\text {gear }} \eta_{\mathrm{FD}} \times i_{g} \times i_{0} \times T_{i}-c_{\mathrm{tm}} \omega_{i}, \\
\omega_{i}=i_{g} \cdot i_{0} \cdot \omega_{\text {wheel }},
\end{gathered}
$$

where $i_{g}$ is the transmission gear ratio, $i_{0}$ is the final drive gear ratio, and $\eta_{\text {gear }}$ and $\eta_{\mathrm{FD}}$ are the transmission and final drive efficiencies, respectively. $T_{i}$ is the transmission input torque, $c_{\mathrm{tm}}$ is the transmission viscous-loss coefficient, $\omega_{i}$ is the transmission input speed, and $\omega_{\text {wheel }}$ is the wheel speed.

The gear-shifting sequence of the AMT is simulated using a discrete-time dynamic model:

$$
\begin{aligned}
& \text { gear }(k+1) \\
& = \begin{cases}9, & \text { gear }(k)+\operatorname{shift}(k)>9, \\
1, & \text { gear }(k)+\operatorname{shift}(k)<1, \\
\operatorname{gear}(k)+\operatorname{shift}(k), & \text { otherwise, }\end{cases}
\end{aligned}
$$

where the state gear is the gear number, and the control shift to the transmission is restricted to take on the values $-1,0$, and 1, corresponding to downshift, sustaining, and up-shift, respectively.

3.1.5. Vehicle Dynamics. It is a common practice that only the vehicle longitudinal dynamics are considered. The longitudinal vehicle dynamics are modeled as a point mass to which various forces are applied:

$$
\omega_{\text {wheel }}(k+1)=\omega_{\text {wheel }}(k)+\frac{T_{\text {wheel }}-T_{\text {brake }}-r_{w}\left(F_{r}+F_{a}\right)}{M_{r} r_{w}^{2}},
$$

where $T_{\text {brake }}$ is the friction brake torque. $F_{r}$ and $F_{a}$ are the rolling resistance and aerodynamic drag forces, respectively. $r_{w}$ is the dynamic tire radius, $M_{r}=M_{v}+J_{r} / r_{w}^{2}$ is the effective mass of the vehicle, and $J_{r}$ is the equivalent moment of inertia of the rotating components in the vehicle.

3.2. DP Problem Formulation. For an optimization problem, choose $u(k)$ to minimize the cost function

$$
J=G_{N}(x(N))+\sum_{k=0}^{N-1} L_{k}(x(k), u(k), w(k)),
$$

where

$$
x(k+1)=f(x(k), u(k), w(k)), \quad k=0,1, \ldots, N-1,
$$

subject to

$$
x(k) \in X(k) \subset \Re^{n}, \quad u(k) \in U(x(k), k) \subset \mathfrak{R}^{m} .
$$


Here, $x(k)$ is the state vector at the stage $k$ in the space of $X(k)$, which contains the transmission gear number gear $(k)$ and battery SOC SOC $(k) ; u(k)$ is the control vector, including the engine throttle throt $(k)$ and the transmission gear shift command $\operatorname{shift}(k) ; w(k)$ is a predetermined disturbance, here it is the rotational speed of the wheel determined by the driving schedule; $f$ is the transition function that represents the system dynamics; $L$ is the instantaneous transition cost; $G_{N}$ is the cost at the final stage $N$.

In this paper, the sampling time for this control problem is 1 second. The cost function to be minimized has the following form:

$$
J=\sum_{k=0}^{N-1}\left[L_{\text {fuel }}(k)+\beta|\operatorname{shift}(k)|\right]+G_{N}\left(x_{\text {SOC }}(N)\right),
$$

where $L_{\text {fuel }}(k)$ is the instantaneous cost of fuel use, $\beta|\operatorname{shift}(k)|$ is adjusted to constrain the vehicle drivability avoiding excessive shifting, and $\beta$ is a positive weighting factor. If there is no constraint on the terminal SOC, the optimization algorithm tends to deplete the battery in order to attain the minimal fuel consumption. Hence, a terminal constraint on SOC $G_{N}\left(x_{\text {SOC }}(N)\right)$ is incorporated into the cost function. During the optimization, it is necessary to impose the following inequality constraints to ensure safe/smooth operation of the engine/battery/motor:

$$
\begin{gathered}
\omega_{e_{-} \text {min }} \leq \omega_{e}(k) \leq \omega_{e_{-} \max } \\
\operatorname{SOC}_{\text {min }} \leq \mathrm{SOC}(k) \leq \operatorname{SOC}_{\max } \\
T_{e_{-} \min }\left(\omega_{e}(k)\right) \leq T_{e}(k) \leq T_{e_{-} \max }\left(\omega_{e}(k)\right) \\
T_{m_{-} \text {min }}\left(\omega_{m}(k)\right) \leq T_{m}(k) \leq T_{m_{-} \max }\left(\omega_{m}(k)\right),
\end{gathered}
$$

where $\omega_{e}$ is the engine speed, SOC is the battery state of charge, and $\mathrm{SOC}_{\min }$ and $\mathrm{SOC}_{\max }$ were selected to be 0.4 and 0.8. $T_{e}$ and $T_{m}$ are the engine and motor torques, respectively.

The DP technique is based on Bellman's Principle of Optimality, which states that the optimal policy can be obtained if we first solve a one-stage subproblem involving only the last stage and then gradually extend to subproblems involving the last two stages, last three stages, and so forth until the entire problem is solved. In this manner, the global dynamic optimization problem can be decomposed into a sequence of the simple minimization problems as follows [15].

Step $N-1$,

$$
\begin{aligned}
J_{N-1}^{*} & (x(N-1)) \\
& =\min _{u(N-1)}[L(x(N-1), u(N-1))+G(x(N))] .
\end{aligned}
$$

Step $k$, for $0 \leq k<N-1$

$$
J_{k}^{*}(x(k))=\min _{u(k)}\left[L(x(k), u(k))+J_{k+1}^{*}(x(k+1))\right],
$$

where $J_{k}^{*}(x(k))$ is the optimal cost-to-go function at the state $x(k)$ starting from the time stage $k$. It represents the optimal resulting cost if the system evolution follows the optimal control law with the current state $x(k)$ from the stage $k$ to the final stage.

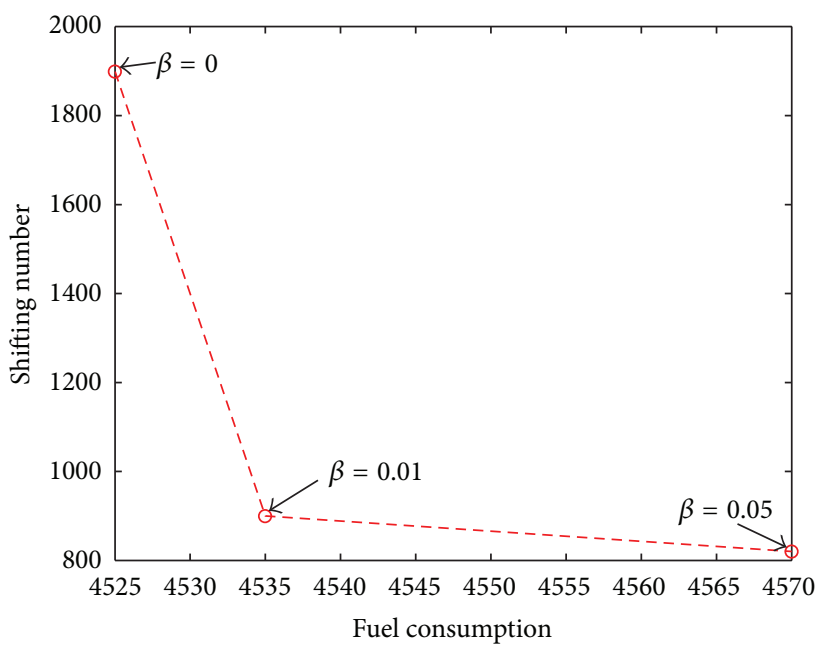

FIGURE 7: Gear-shifting number versus fuel consumption.

The foregoing recursive equations are firstly solved backward and then searched forward to find the optimal control policy. The minimization is performed subject to the inequality constraints shown in (14).

\section{Development of Improved Control Strategy}

4.1. DP Results. The DP procedure described above produces an optimal solution with respect to (13), where the shift weighting factor $\beta$ was selected to constrain the AMT shift number. It can be found that a larger value of $\beta$ results in less frequent gear shifting, but the larger fuel consumption. The balance relationship of the gear shifting number and the fuel consumption is shown in Figure 7, and $\beta=0.01$ is chosen for the subsequent analysis.

Simulation results of the vehicle based on the DP optimization are shown in Figure 8. The AMT shifts between the 4th and the 9th gear ratio mostly. The SOC increases near 0.6 at the end of the driving cycle due to the penalty in the cost function shown in (13). The power of the engine increases after $1900 \mathrm{~s}$ apparently to make SOC a rise. It can be seen that most of the engine operating points fall in the highefficiency area, near the optimal fuel consumption line before $1400 \mathrm{rpm}$ and near the maximum torque line after $1400 \mathrm{rpm}$. Compared to the preliminary rule-based control strategy, the fuel economy under the DP optimal control law is improved by $15.9 \%$.

4.2. Development of Improved Control Strategy. The DP control policy is not applicable in real driving conditions in that it requires knowledge of the future speed and load profiles. However, analyzing carefully the DP-based results is able to help us in improving the preliminary rule-based control strategy.

Firstly, we study how the power split based on the preliminary control strategy can be improved. A power-split ratio PSR $=P_{\text {eng }} / P_{\text {req }}$ is defined to quantify the positive power flow in the powertrain, where $P_{\text {eng }}$ is the engine power and 


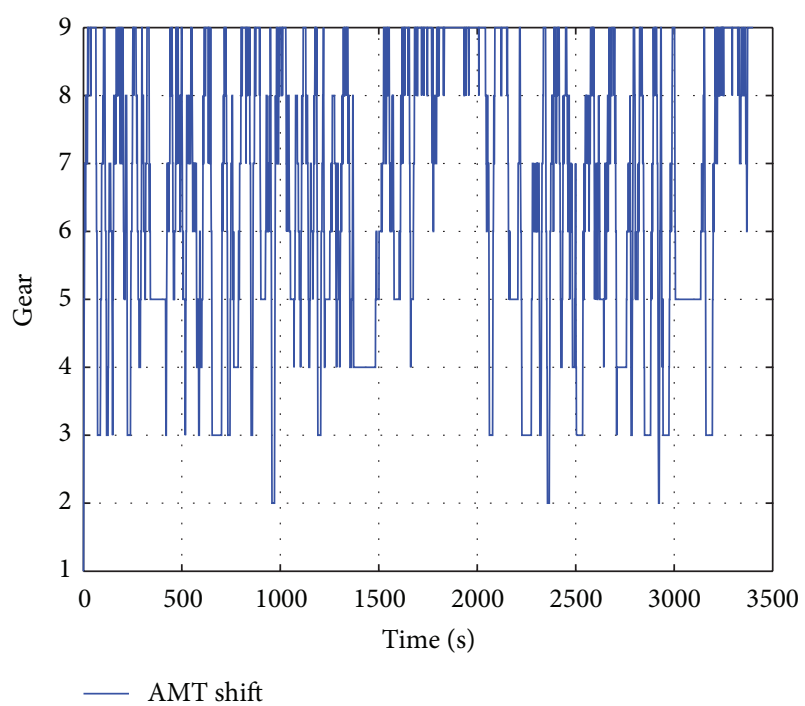

(a)

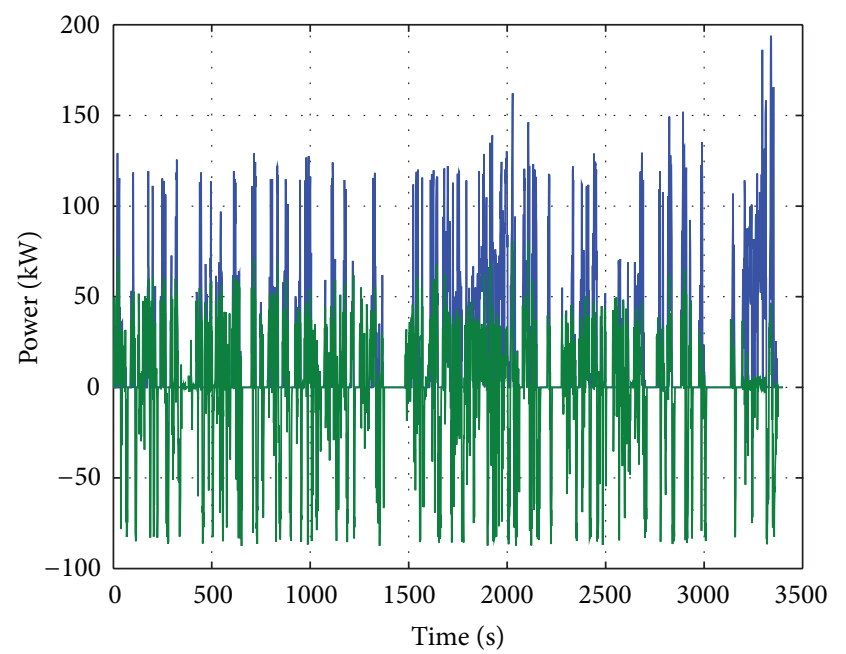

Engine power $(\mathrm{kW})$

_ Battery power $(\mathrm{kW})$

(c)

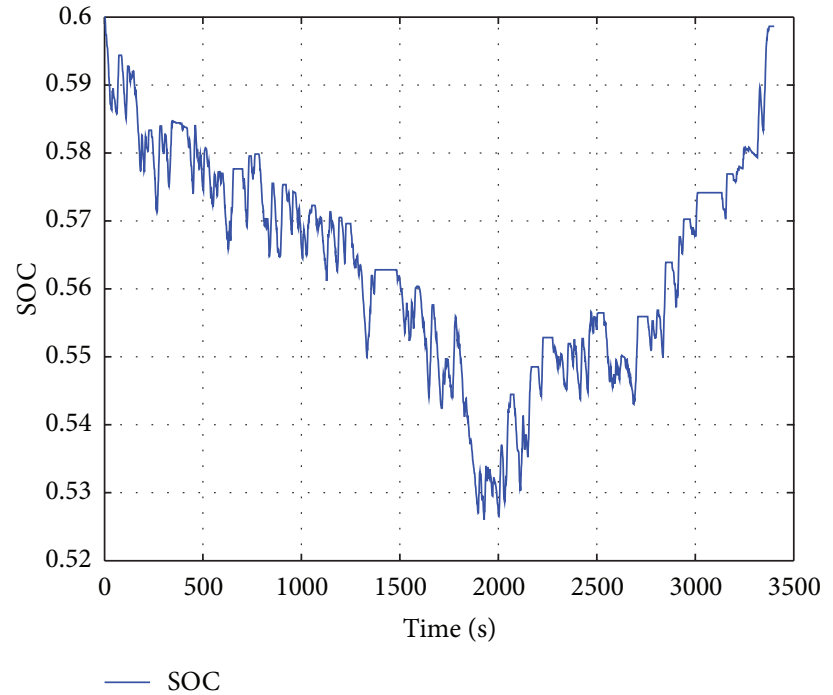

(b)

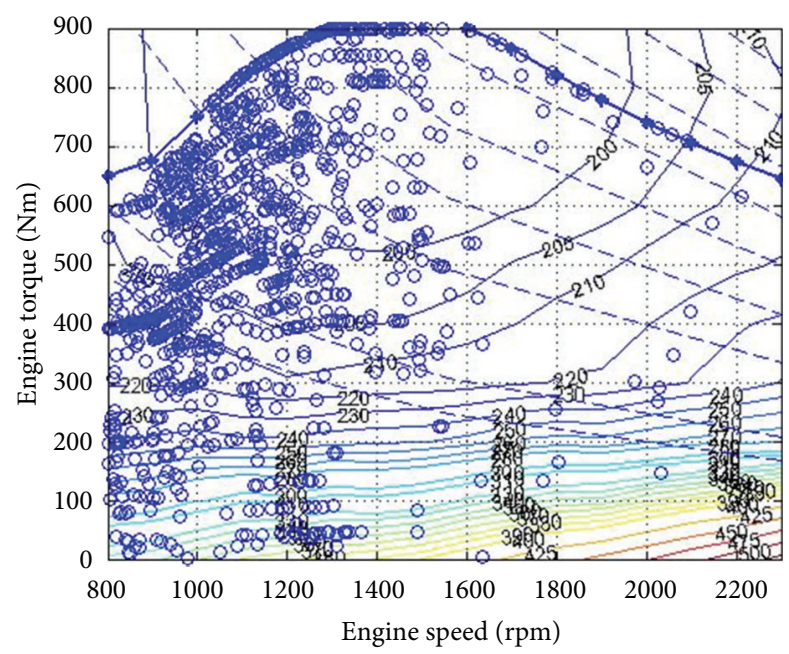

(d)

FIGURE 8: DP-based results.

$P_{\text {req }}$ is the power requested. Four positive-power operating modes are defined:

(1) PSR = 0: motor-only mode;

(2) $\mathrm{PSR}=1$ : engine-only mode;

(3) $0<$ PSR < 1: power-assist mode;

(4) PSR > 1: recharging mode.

The enhanced rule can be found by plotting the optimal PSR as a function of the torque demand at transmission input, as shown in Figure 9. It is remarkable that the optimal policy uses the recharging mode in the low torque region, the engine-only mode in the middle torque region, and the power-assist mode in the high torque region. In order to extract a realizable rule, the least-square curve fit is deployed to approximate the optimal PSR points. The red line in Figure 8 shows the fitting result.

The gear shifting schedule is crucial to the fuel economy of hybrid electric vehicles. In the DP scheme, the gearshift command is one of the control variables. The gear operating points from the DP-based result are plotted in the form of the power demand versus vehicle speed (see Figure 10). It can be seen that the gear positions are separated into different regions, and the boundaries between adjacent regions represents the optimal gear shifting thresholds. The gear shifting schedule can thus be obtained on the basis of these thresholds. 


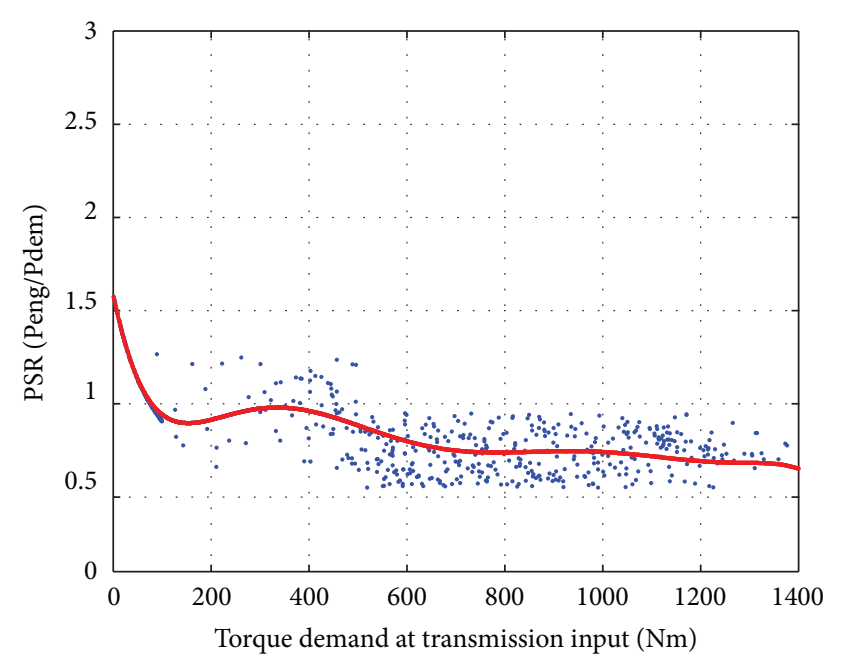

- Optimal operating points (DP)

- Fitted optimal PSR line

FIGURE 9: DP-based power split behavior.

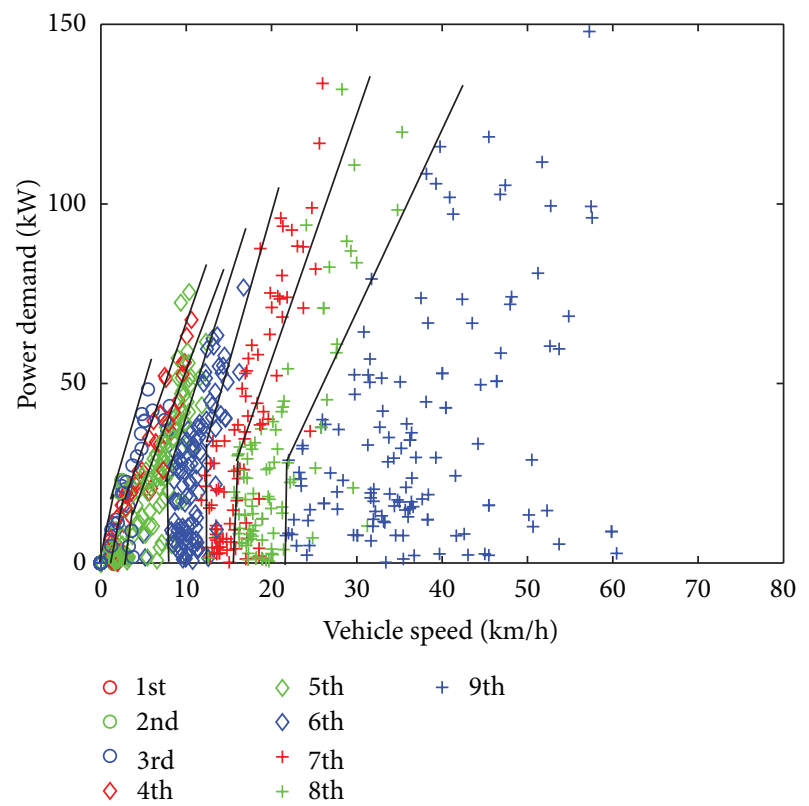

FIGURE 10: DP-based gear operating points.

According to the extracted rules, the improved rulebased control strategy is designed and implemented in the MATLAB/Stateflow environment.

4.3. Fuel Economy Evaluation. The preliminary and improved rule-based control strategies, as well as the benchmark, the DP control policy, are compared by means of the nature driving schedule from the Weichai Power Company Limited. The results are shown in Table 2. It is clear that the improved rule-based control strategy extracted from the DP-based results can ensure a much better fuel economy for the hybrid electric truck, compared with the preliminary one.
TABle 2: Fuel economy comparison.

\begin{tabular}{lcc}
\hline & $\begin{array}{c}\text { Fuel economy } \\
\text { (mile per US gallon) }\end{array}$ & Improvement \\
\hline Preliminary rule based & 6.78 & - \\
Improved rule based & 7.45 & $9.9 \%$ \\
Hybrid truck (DP) & 7.86 & $15.9 \%$ \\
\hline
\end{tabular}

\section{Conclusions}

A feed-forward model of a heavy-duty parallel hybrid electric truck is established for studying the optimal energy management strategy. The preliminary rule-based control strategy is designed based on the static optimization algorithm. Based on the simplified model, DP is applied to solve the globally optimal energy control strategy. Improved rule-based control rules that are applicable in real time are extracted from analyzing the DP-based results. Based on a natural driving schedule from Weichai Power Company Limited, a comparison is conducted between the improved rule-based control strategy and the preliminary one derived from static optimization. The result demonstrates that the improved rule-based control policy from the DP algorithm results in a noteworthy enhancement on the fuel economy of the hybrid electric truck. The proper approximation of DP behavior, however, is the key process to extract the control rule and is worth further investigation.

\section{Acknowledgments}

The authors would like to express their deep gratitude to Professor Huei Peng in The University of Michigan for many helpful suggestions on the DP algorithm. This work was supported by the Natural Science Foundation of China (50905015).

\section{References}

[1] B. M. Baumann, G. Washington, B. C. Glenn, and G. Rizzoni, "Mechatronic design and control of hybrid electric vehicles," IEEE/ASME Transactions on Mechatronics, vol. 5, no. 1, pp. 58$72,2000$.

[2] N. J. Schouten, M. A. Salman, and N. A. Kheir, "Fuzzy logic control for parallel hybrid vehicles," IEEE Transactions on Control Systems Technology, vol. 10, no. 3, pp. 460-468, 2002.

[3] A. Sciarretta and L. Guzzella, "Control of hybrid electric vehicles," IEEE Control Systems Magazine, vol. 27, no. 2, pp. 60-70, 2007.

[4] G. Paganelli, G. Ercole, A. Brahma, Y. Guezennec, and G. Rizzoni, "A general formulation for the instantaneous control of the power split in charge-sustaining hybrid electric vehicles," in Proceedings of the 5th International Symposium on Advanced Vehicle Control (AVEC '00), Ann Arbor, Minn, USA, 2000.

[5] V. H. Johnson, K. B. Wipke, and D. J. Rausen, "HEV control strategy for real-time optimization of fuel economy and emissions," in Proceedings of the Future Car Congress, SAE paper no. 2000-01-1543. 
[6] A. Brahma, Y. Guezennec, and G. Rizzoni, "Dynamic optimization of mechanical electrical power flow in parallel hybrid electric vehicles," in Proceedings of the 5th International Symposium on Advanced Vehicle Control (AVEC '00), Ann Arbor, Minn, USA, 2000.

[7] U. Zoelch and D. Schroeder, "Dynamic optimization method for design and rating of the components of a hybrid vehicle," International Journal of Vehicle Design, vol. 19, no. 1, pp. 1-13, 1998.

[8] C. C. Lin, J. M. Kang, J. W. Grizzle, and H. Peng, "Energy management strategy for a parallel hybrid electric truck," in Proceedings of the American Control Conference, pp. 2878-2883, Arlington, Va, USA, June 2001.

[9] J. M. Miller, Propulsion Systems for Hybrid Vehicles, The Institution of Engineering and Technology, Stevenage, UK, 2004.

[10] S.-J. Hou, Y. Zou, and R. Chen, "Feed-forward model development of a hybrid electric truck for power management studies," in Proceedings of the 2nd Intelligent Control and Information Processing (ICICIP '11), Harbin, China, 2011.

[11] J. Liu, Modeling, configuration and control optimization of powersplit hybrid vehicles [Ph.D. thesis], University of Michigan, 2007.

[12] D. L. McKain, N. N. Clark, T. H. Balon, P. J. Moynihan, S. A. Lynch, and T. C. Webb, "Characterization of emissions from hybrid-electric and conventional transit buses," SAE Paper 2000-01-2011, 2000.

[13] Society of Automotive Engineers, "Recommended Practice for Measuring Exhaust Emissions and Fuel Economy of HybridElectric Vehicles," Hybrid-Electric Vehicle Test Procedure Task Force SAE J1711, 1998.

[14] I. Kolmanovsky, M. van Nieuwstadt, and J. Sun, "Optimization of complex powertrain systems for fuel economy and emissions," in Proceedings of the IEEE International Conference on Control Applications (CCA '99), pp. 833-839, August 1999.

[15] R. Bellman, Dynamic Programming, Princeton Landmarks in Mathematics, Princeton University Press, Princeton, NJ, USA, 2010. 


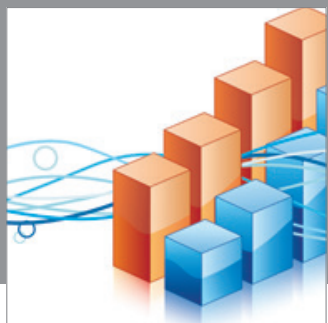

Advances in

Operations Research

mansans

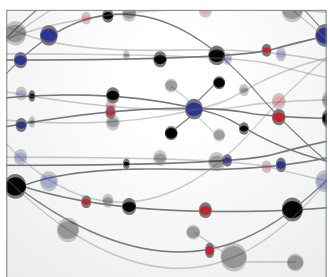

The Scientific World Journal
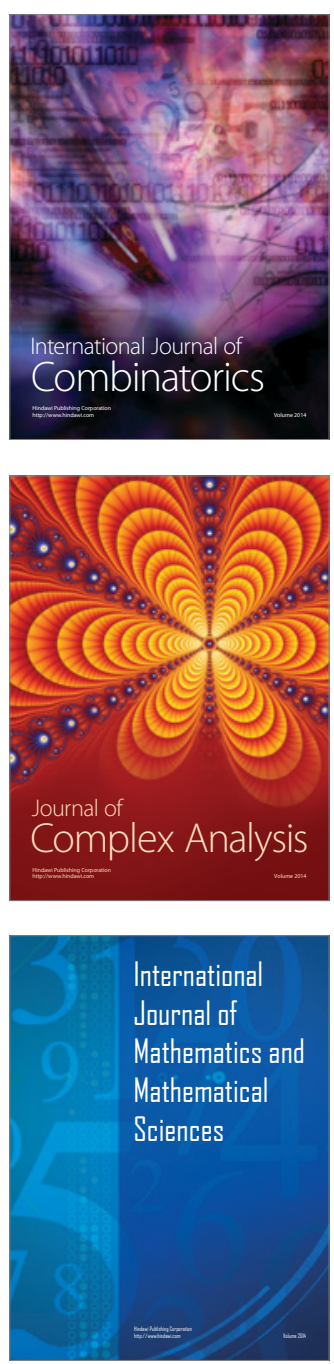
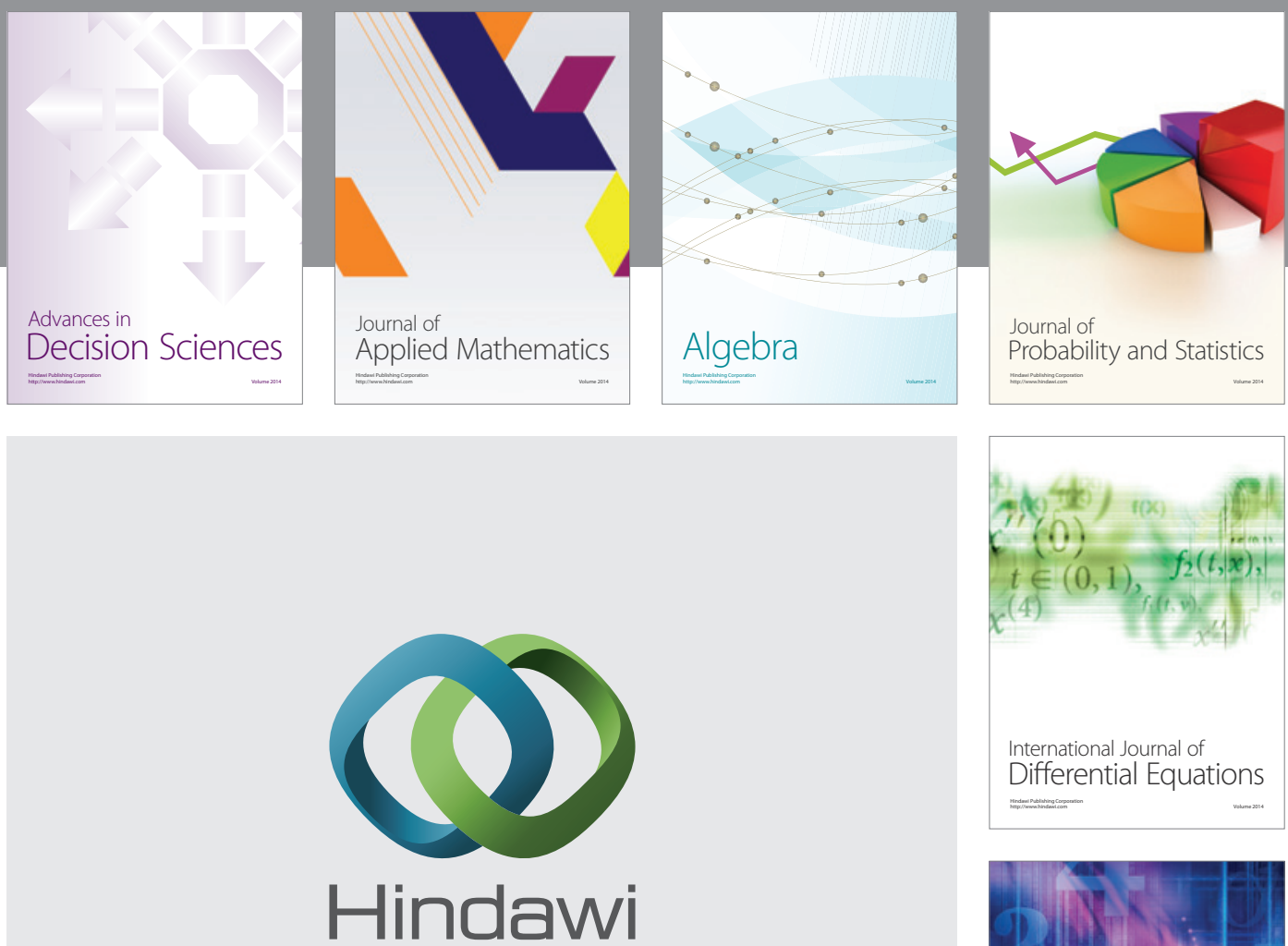

Submit your manuscripts at http://www.hindawi.com
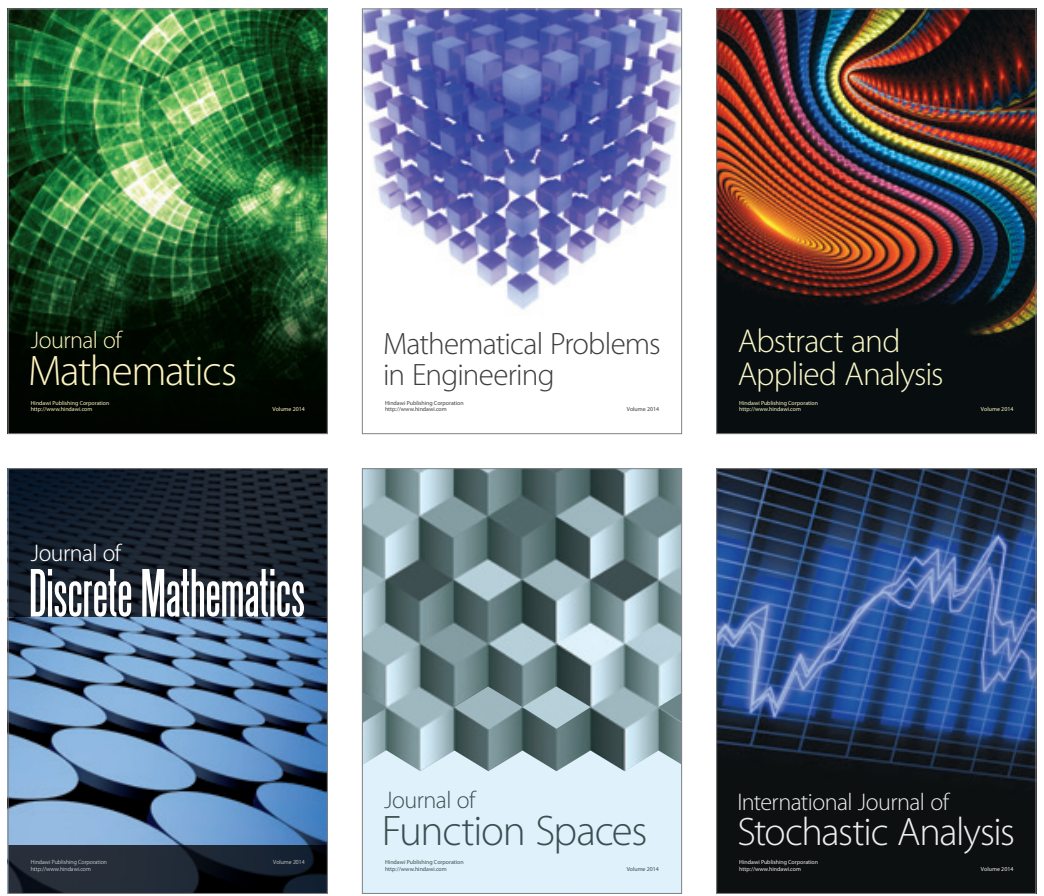

Journal of

Function Spaces

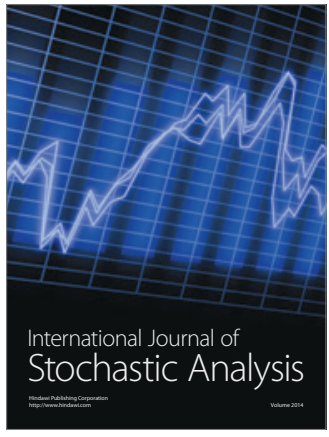

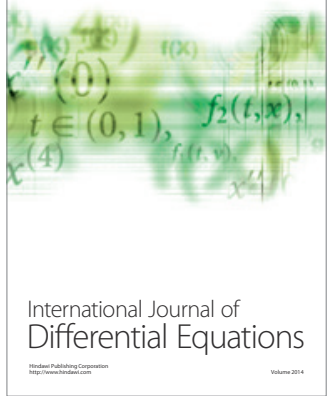
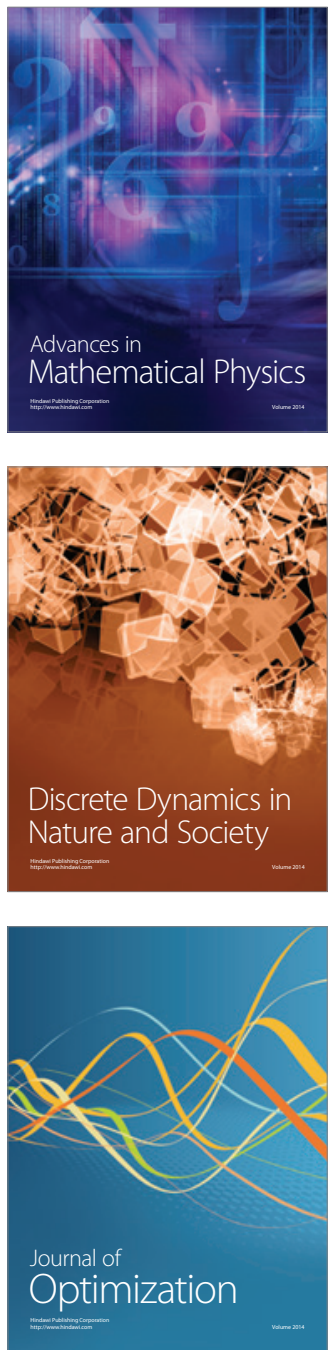